\title{
Low Anterior Resection Syndrome in Adults with Rectal Cancer in China: a Case Series Analysis
}

\author{
Yuan Qiu $^{1} \cdot$ Yu Pu $^{1} \cdot$ Haidi Guan ${ }^{1} \cdot$ Weijie Fan ${ }^{2}$. Shuai Wang ${ }^{1} \cdot$ Guangsheng Du ${ }^{1} \cdot$ Hua Yang ${ }^{1} \cdot$ Weidong Xiao $^{1}$
}

Received: 3 August 2020 / Accepted: 15 January 2021 / Published online: 28 January 2021

(C) The Author(s) 2021

\begin{abstract}
Low anterior resection syndrome (LARS) comprises a collection of symptoms affecting patients' defecation after restorative surgery for rectal cancer. The aim of this work was to study the incidence and risk factors for LARS in China. Rectal cancer patients undergoing total mesorectal excision and colorectal anastomosis between May 2012 and January 2015 were identified from a single center. The patients completed the LARS score questionnaire through telephone. The clinical and pathological factors that may influence the occurrence of LARS were analyzed using univariate and multivariate logistic regression analysis. The influence of postoperative recovery time and pelvic dimensions on the occurrence of LARS was also analyzed. This study included 337 patients, at an average age of 61.03 SD11.32. The mean LARS score of the patients was 14.08 (range 0-41). A total of 126 patients (37.4\%) developed LARS after surgery, including $63(18.7 \%)$ severe cases. Compared with the scores within the initial 6 postoperative months, the LARS scores of the patients in $6 \sim 18$ months after the surgery showed significant reductions $(p<0.01)$. In multivariate analysis, lower locations of anastomosis, pre-surgery radiotherapy, and shorter postoperative recovery time were significant predisposing factors for LARS. A subgroup analysis revealed that patients suffering from LARS over 18 months after surgery were found to have a significantly shorter interspinous distance than those without LARS $(p<0.05)$. LARS could improve over time after surgery. Lower anastomotic level and pre-surgery radiotherapy are risk factors for LARS.
\end{abstract}

Keywords LARS $\cdot$ Rectal cancer $\cdot$ Surgery $\cdot$ Pathological analysis

\section{Introduction}

As a significant cause of cancer death worldwide, colorectal cancer (CRC) is still one of the most common cancers in the world. The annual incidence of rectal cancer in China exceeds 200,000 cases [1]. The combination of advances in surgical technique and neoadjuvant therapy have led to an increase in sphincter preservation. Low anterior resection (LAR) with total mesorectal excision (TME) is currently regarded as the optimal procedure for the majority of patients with rectal cancer. However, many patients undergoing LAR suffer from

Yuan Qiu and Yu Pu contributed equally.

Weidong Xiao

weidongxiao2@163.com

1 Department of General Surgery of Xinqiao Hospital, the Army Medical University, Shapingba, Chongqing 400037, China

2 Department of Radiology of Xinqiao Hospital, the Army Medical University, Shapingba, Chongqing 400037, China bowel dysfunction. The complex of symptoms consisting of incontinence for flatus and/or feces, urgency, constipation, fragmentation, and frequent bowel movements is referred to as the low anterior resection syndrome (LARS) [2]. Even though recognized for a long time, this disorder has not been systematically studied. Therefore, anorectal function after sphincter-preserving surgery has not received enough attention in both research and clinical practice.

The broad definition of LARS has made it difficult to assess the quality of life of patients, and comparison of outcomes between patients has been challenging. A score has been developed on the basis of patient-reported symptoms, which is called the LARS score [2]. This score is a quick simple and valid questionnaire for measuring the severity of postoperative bowel dysfunction. In this survey, five issues that most bothered the patients were selected: incontinence for flatus, incontinence for liquid stool, frequency, clustering, and urgency. It is a summative scale, and each item is individually weighted. Developed in Danish, the LARS score has been validated and translated into several languages, including Chinese [3-5]. Although numerous studies have focused on 
the association between quality of life and LARS score, very few studies have used the LARS score to predict risk factors for postoperative bowel dysfunction, especially in China [6].

The incidence of LARS after LAR greatly varies in the literature [7]. The symptoms were previously thought to be transient. Recent study reported that up to $30 \%$ of LAR patients have ongoing symptoms for more than 1 year [8]. Like the symptom profile, the etiology of LARS is difficult to define and is likely related to multifactorial mechanisms: sphincter injury during anastomosis, alterations in anorectal physiology, disappearance of the rectoanal inhibitory reflex, and reduction in rectal reservoir capacity and compliance [9]. Therefore, it is important to identify the patient's factors that predispose to LARS. Moreover, detection of these factors associated with LARS should have a great value in helping doctors, and their patients make better decisions about choice of the kind of surgery. The main aims of this study were to investigate the prevalence of LARS as well as to evaluate the predictive factors of LARS using a validated scoring system.

\section{Patients and Methods}

\section{Study Population}

This study was approved by the Research and Ethics Committee of Xinqiao Hospital, the Army Medical University (2015-YD037-02). Patients were identified from a rectal cancer database at our single center. Patient details were retrieved from a maintained departmental database in our center (from May 2012 to January 2015). All participants gave their written informed consent. Inclusion criteria included the following: (1) patients who were diagnosed with rectal cancer and had undergone sphincter-preserving low anterior resection with curative intent and (2) age 18 years or older. Exclusion criteria were intestinal stoma, intestinal obstruction, recurrence, other cancers, intellectual disability/dementia, and metastatic disease. Demographic and clinical information was obtained from databases. In total, 536 eligible patients were identified, of whom 199 were excluded (Fig. 1). Patient's age, gender, preoperative chemoradiotherapy, surgical procedure, morbidity, anastomosis level, and pathological features were retrieved from case records. Pelvic dimensions, including interspinous distance, transverse outlet, sagittal inlet, posterior sagittal diameter of pelvic outlet, and sacrum-coccyx distance, were blindly measured on three-dimensional medical CT image reconstruction by a radiologist (W.F.).

\section{Follow-Up}

We conducted this descriptive and exploratory study using a telephone-based survey with LARS questionnaire. Also, questionnaire survey was conducted during outpatient follow-up.

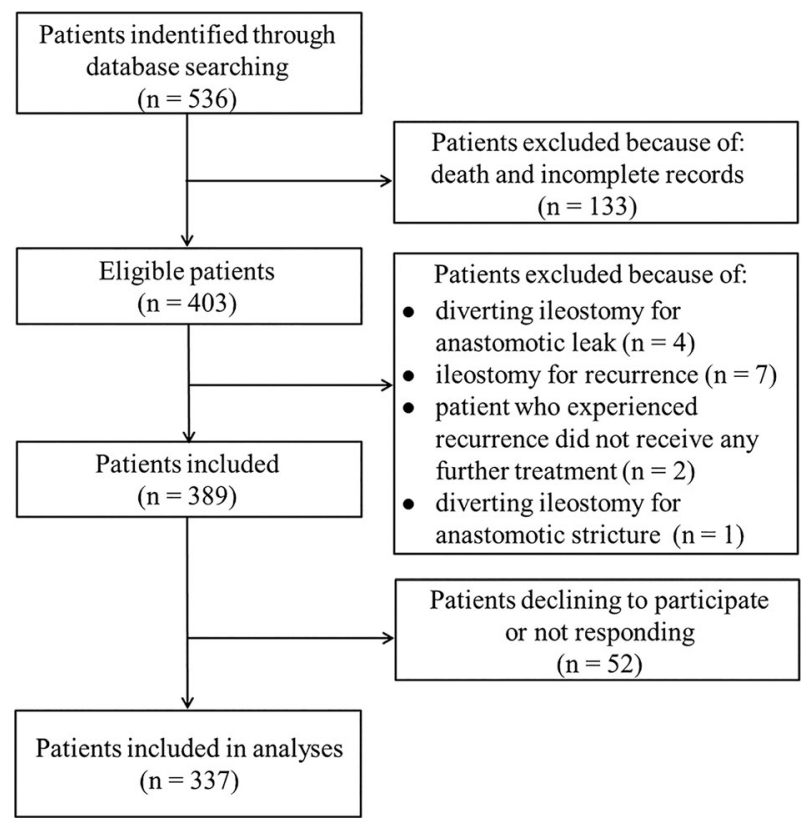

Fig. 1 Flow chart of patient selection

There was no statistical difference in the survey results whether based on the outpatient follow-up or on the telephone. The surveys were conducted at least 30 days past surgery, and, hence, their bowel function was expected to have regained stability. Each question received a score based on the reply, and the scores for each question are then added together to give a final score range of $0-42$. The LARS score was categorized into 3 groups: no LARS (0-20 points), minor LARS (21-29 points), or major LARS (30-42 points).

\section{Statistical Analysis}

Data on quantitative variables are presented as mean and standard deviation, and frequencies for qualitative variables. The patients selected were grouped into two groups: group I consists of patients with major/mild LARS and group II consists of patients with no LARS. A Chi-square test was applied to assess differences between the study groups for qualitative parameters. We used the Mann-Whitney $U$-test for continuous data and the $\chi^{2}$ test or Fisher's exact test for categorical data. Univariate analysis was carried out and compared the presence or absence of LARS (yes/no) with patient and tumorrelated variables. Multivariate analysis was performed of those variables which showed a statistically significant association on univariate analysis of $p<0.05$.

\section{Results}

We identified 536 patient records. Of these, we excluded 199 for the following reasons: incomplete or missing records or death 
$(n=133)$, enterostomies due to anastomotic leakage $(n=4)$, cancer recurrence $(n=9)$, ileostomy due to anastomotic stricture $(n=1)$, and loss of follow-up ( $n=52)$ (Fig. 1). Therefore, this study population included 337 patients, 208 men and 129 women. At the time of the surgery, the mean age of all patients was 61.03 SD11.32 years. The mean LARS score from all participants was 14.08 (range, 0-41). There were 211 (62.6\%) patients with no LARS, 63 (18.7\%) with minor LARS and 63 (18.7\%) with major LARS. LARS was significantly more frequent in patients with lower locations of anastomosis $(58.8 \%$ vs. $23.8 \%$, $p<0.05$ ), in those with preoperative radiotherapy $(83.3 \%$ vs. $35.7 \%, p<0.05)$, and those who had shorter postoperative recovery time $(p<0.05)$ (Table 1$)$. The patients were divided into 4 groups according to the time to complete the questionnaire: $1 \sim 3$ months $(n=42)$; 3 6 months $(n=61) ; 6 \sim 18$ months $(n=$ $132)$; and more than 18 months $(n=102)$. The analysis about the relationship between the postoperative recovery time and LARS score revealed that compared with the patients in the first 6 postoperative months, the LARS score of the patients in the period of 6-18 postoperative months displayed a significant gradual decrease trend $(p<0.01)$ (Fig. 2). In comparing the LARS scores between the 1 3 months and 3 6 months, there is no significant difference.

The multivariate analysis model, including lower locations of anastomosis, pre-surgery radiotherapy, and shorter postoperative recovery time, was significantly associated with LARS. Statistical analysis showed that patients of presurgery radiotherapy had nearly 13 -fold of increased risk of developing LARS $(p<0.01)$ and patients of lower locations of anastomosis had nearly 5.3-fold of increased risk of developing LARS $(p=0.03)$ (Table 2). Moreover, shorter postoperative recovery time independently associated with LARS.

Based on above data, as many as $22.5 \%$ of patients still have LARS for more than 18 months after surgery. Thus, we investigate the clinical characteristics of patients who underwent previous surgery more than 18 months. Low rectal cancer patients (with an anastomotic level $<5 \mathrm{~cm}$ ) had a higher incidence of LARS $(p<0.05)$.

To further study the relationship between surgical difficulty and LARS, pelvic dimensions from patients who had an anastomotic level $<5 \mathrm{~cm}$ and underwent surgery more than 18 months were utilized to analysis. Interspinous diameters of LARS group were significantly lower than No LARS group $(p<0.05)$ (Table 3). However, other variables such as transverse outlet, sagittal inlet, posterior sagittal diameter of pelvic outlet, and sacrum-coccyx distance were not found to be statistically significant.

\section{Discussion}

The present study is the first attempt in China to investigate the prevalence and risk factors for LARS using a scoring system. This study revealed that $22.5 \%$ of patients still have some degree of LARS for more than 18 months after surgery. Moreover, our study highlighted that lower locations of anastomosis, pre-surgery radiotherapy, and shorter postoperative recovery time are the independent risk factors of LARS occurrence. The interspinous diameter may be a potential predicating factor in LARS recovery of the patients with low rectal cancer.

It is undeniable that most rectal cancer patients will experience daily bowel dysfunction after LAR. Patients may have a combination of symptoms including frequency, urgency, incontinence, and constipation. This important realization has led to the development of a simple self-administered scoring system assessing the severity of LARS - the LARS score [2]. Prevalence of LARS assessed by this system varies in the literature between 40 and $60 \%$, including studies with short follow-up (12 months) and longer follow-up (up to 14 years) $[10,11]$. For the majority of patients, symptoms of LARS will decrease over time. In this study, $37.4 \%$ of patients developed LARS after the surgery, and as time went by, $22.5 \%$ of patients still experienced LARS symptoms for up to 18 months following surgery. The incidence of LARS in this study was slightly lower than in other studies. One possible explanation for this was that the proportion of preoperative radiotherapy in this study was lower than that published by other studies. It is recognized that preoperative radiotherapy had an influence on the anorectal function. Notably, only about $3.6 \%$ (12 of 337) of patients received preoperative radiotherapy in our study. Other studies about LARS have demonstrated that up to 20 to $70 \%$ of patients received pre-surgery radiotherapy $[12,13]$. There are a lot of reasons for such a low rate of pre-surgery radiation therapy in our center. One reason is that the level of understanding and acceptance of pre-surgery radiation among Chinese people is relatively low, especially in economically backward west areas.

Several factors have been reported to be associated with LARS including age, gender, operation method (laparotomy vs. laparoscopy), T stage, level of anastomosis, radiotherapy, and pelvic cavity. Different studies have provided different conclusions. In this study, gender was not associated with LARS. Bregendahl et al. reported that female gender was a risk factor for LARS [12], while some authors found that male was related to LARS [14]. On the other hand, LARS prevalence has been reported to be lower in older patients compared to younger patients $[12,15]$. However, we did not find any statistical difference in our study. The only factors related to LARS in this study were as follows: preoperative radiotherapy, level of anastomosis, and shorter postoperative recovery time. Among these factors, preoperative radiotherapy has been the most extensively studied. It does cause tissue edema, fibrosis, extensive mist, and exudates which impede the dissection of the tissue [16]. Luis et al. reported that both preoperative radiotherapy and postoperative radiotherapy were risk 
Table 1 Association analysis between clinical parameters and the occurrence of low anterior resection syndrome (LARS)

\begin{tabular}{|c|c|c|c|}
\hline Patient demographics & $\begin{array}{l}\text { No } \\
\text { LARS }\end{array}$ & $\begin{array}{l}\text { LARS } \\
\text { (major/ } \\
\text { minor) }\end{array}$ & $\begin{array}{l}p \\
\text { value }\end{array}$ \\
\hline All & 211 & 126 & \\
\hline \multicolumn{4}{|l|}{ Sex } \\
\hline Male & 131 & 77 & \multirow[t]{2}{*}{0.859} \\
\hline Female & 80 & 49 & \\
\hline \multicolumn{4}{|l|}{ Age } \\
\hline$<60$ years & 82 & 53 & \multirow[t]{2}{*}{0.562} \\
\hline$\geq 60$ years & 129 & 73 & \\
\hline \multicolumn{4}{|l|}{ Surgical treatment } \\
\hline Laparotomy & 24 & 10 & \multirow[t]{2}{*}{0.311} \\
\hline Laparoscopy & 187 & 116 & \\
\hline \multicolumn{4}{|l|}{ T stage } \\
\hline $\mathrm{T} 0 \sim \mathrm{T} 2$ & 96 & 53 & \multirow[t]{2}{*}{0.539} \\
\hline $\mathrm{T} 3 \sim \mathrm{T} 4$ & 115 & 73 & \\
\hline \multicolumn{4}{|l|}{$\mathrm{N}$ stage } \\
\hline N0 & 125 & 70 & \multirow[t]{2}{*}{0.507} \\
\hline $\mathrm{N} 1 \sim \mathrm{N} 2$ & 86 & 56 & \\
\hline \multicolumn{4}{|l|}{ Anastomosis locations } \\
\hline$<5 \mathrm{~cm}$ & 54 & 77 & \multirow[t]{2}{*}{0.000} \\
\hline$\geq 5 \mathrm{~cm}$ & 157 & 49 & \\
\hline \multicolumn{4}{|l|}{ Pre-surgery radiotherapy } \\
\hline No & 209 & 116 & \multirow[t]{2}{*}{0.002} \\
\hline Yes & 2 & 10 & \\
\hline \multicolumn{4}{|l|}{ Follow-up duration } \\
\hline 1 month 3 months & 18 & 24 & \multirow[t]{4}{*}{0.000} \\
\hline 3 month $\sim 6$ months & 30 & 31 & \\
\hline 6 month $\sim 18$ months & 84 & 48 & \\
\hline$>18$ months & 79 & 23 & \\
\hline \multicolumn{4}{|c|}{$\begin{array}{l}\text { Posterior sagittal diameter of pelvic } \\
\text { outlet }\end{array}$} \\
\hline$<90 \mathrm{~mm}$ & 151 & 82 & \multirow[t]{2}{*}{0.212} \\
\hline$\geq 90 \mathrm{~mm}$ & 60 & 44 & \\
\hline \multicolumn{4}{|l|}{ Transverse outlet } \\
\hline$<108.5 \mathrm{~mm}$ & 108 & 61 & \multirow[t]{2}{*}{0.622} \\
\hline$\geq 108.5 \mathrm{~mm}$ & 103 & 65 & \\
\hline \multicolumn{4}{|l|}{ Interspinous distance } \\
\hline$<96.2 \mathrm{~mm}$ & 104 & 69 & \multirow[t]{2}{*}{0.331} \\
\hline$\geq 96.2 \mathrm{~mm}$ & 107 & 57 & \\
\hline \multicolumn{4}{|l|}{ Sagittal inlet } \\
\hline$<104 \mathrm{~mm}$ & 114 & 61 & \multirow[t]{2}{*}{0.318} \\
\hline$\geq 104 \mathrm{~mm}$ & 97 & 65 & \\
\hline \multicolumn{4}{|l|}{ Sacrum-coccyx distance } \\
\hline$<119 \mathrm{~mm}$ & 105 & 62 & \multirow[t]{2}{*}{0.921} \\
\hline$\geq 119 \mathrm{~mm}$ & 106 & 64 & \\
\hline
\end{tabular}

factors associated with LARS [17]. It is possible that for some of these irradiated patients with low rectal cancer, a sphincter-

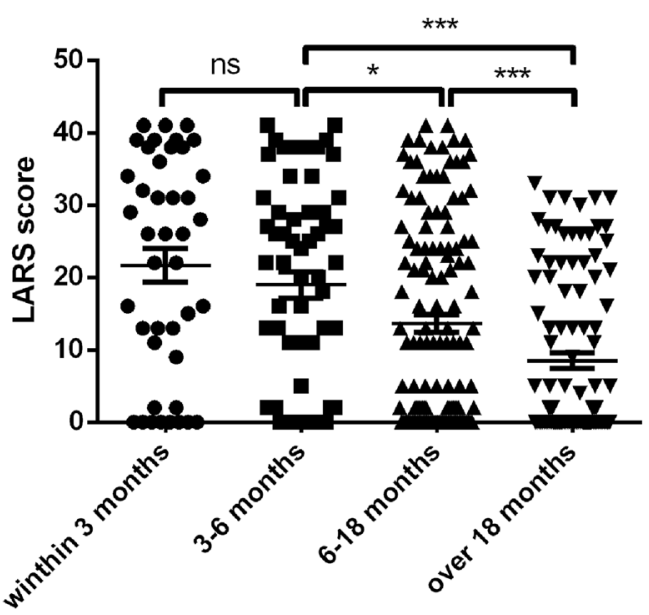

Fig. 2 Distribution of low anterior resection scores according to postoperative recovery time

preserving surgery was not the optimal procedure, and the abdominoperineal resection should also be considered. These findings emphasize the need of an intricate decisionmaking to balance rectal cancer staging, patient beliefs, and surgeon-related factors.

Another factor found to correlate with LARS is the level of anastomosis. In other literatures, the levels of anastomosis related to LARS ranged from 2 to $12 \mathrm{~cm}[18,19]$. Recent study found that the best cut-off value for LARS was $5 \mathrm{~cm}$ (anastomosis level), which corresponded to a tumor level of around $7 \mathrm{~cm}$ from the anal verge $[13,20]$. Thus, the cut-off point was set at $5 \mathrm{~cm}$ in this study. We found a 5.3 -fold increase in the risk of LARS in patients who had lower locations of anastomosis $(<5 \mathrm{~cm})$. This is consistent with the findings from the other studies, where pronounced bowel dysfunction was reported for patients with low-lying tumors. Interestingly, LARS occurred not only in rectal cancer cases who underwent low anterior resection but also in patients with a high-lying tumor (locations of anastomosis $>5 \mathrm{~cm}$ ) who had anterior resection, although at a lower frequency (23.8\%). Understanding the pathophysiology of such failures of functional recovery may help in surgical decision-making and anorectal rehabilitation for rectal cancer patients. In this respect, some studies suggest that the type of reconstruction methods, such as side-to-end anastomosis and colonic Jpouch, used for bowel anastomosis influence the functional outcome [21-23]. Unfortunately, the differences in LARS

Table 2 Univariate analysis of factors associated with the risk of developing low anterior resection syndrome

\begin{tabular}{llll}
\hline Variable & OR & $95 \% \mathrm{CI}$ & $\mathrm{p}$ \\
\hline Location of anastomosis & 5.336 & $3.197 \sim 8.907$ & 0.000 \\
Pre-surgery radiotherapy & 13.216 & $2.597 \sim 67.260$ & 0.002 \\
Postoperative recovery time & 3.477 & $2.033 \sim 5.946$ & 0.000 \\
\hline
\end{tabular}


Table 3 Comparison of pelvic parameters in patients with and without low anterior resection syndrome who had an anastomotic level $<5 \mathrm{~cm}$ and underwent surgery more than 18 months

\begin{tabular}{lllllll}
\hline & $\mathrm{n}$ & Interspinous distance & Transverse outlet & Sagittal inlet & $\begin{array}{l}\text { Posterior sagittal diameter } \\
\text { of pelvic outlet }\end{array}$ & Sacrum-coccyx distance \\
\hline No LARS & 29 & $100.97 \pm 10.28$ & $113.37 \pm 13.48$ & $104.67 \pm 13.74$ & $84.88 \pm 8.08$ & $116.90 \pm 16.19$ \\
LARS & 19 & $94.04 \pm 8.71^{*}$ & $105.40 \pm 14.61$ & $104.20 \pm 9.61$ & $85.21 \pm 8.68$ & $118.26 \pm 13.63$ \\
\hline
\end{tabular}

$*, P<0.05$

score after different types of anastomotic technique have not been evaluated in this study due to incomplete records.

There is a correlation between LARS and postoperative recovery time which was confirmed in the present study. It has been reported and it is also our clinical experience that bowel dysfunction stabilizes within the first 1-2 years, although some patients experience poor anorectal function within 6 months after LAR. This study found that some patients show an improvement of anorectal function at 18 months after surgery. Moreover, long-term studies show minimal functional differences overall over a 2-year or greater follow-up [8]. The results presented above suggest that the cause of LARS is considered to be multifactorial, including damage to the anatomy or the function of the anal sphincters, neurologic impairment, and poor neorectal capacity. Among them, some deficits are considered recoverable. For example, the hypogastric nerves could be injured by the dissection of the rectum, and consequently the rectoanal inhibitory reflex (RAIR) is absent postoperatively. In this respect, O'Riordain et al. have demonstrated that the RAIR disappeared in $83 \%$ of patients with LAR, with RAIR recovery at 6 months in $21 \%$ and in $85 \%$ at 24 months [24]. Consistent with this, some investigators found that autonomic nerves regeneration occur across the anastomotic scar 6 months after surgery [25]. However, some deficits are considered unrecoverable. The most obvious mechanism potentially responsible for LARS is the distension of the sphincter muscles after the insertion of the circular stapling device. In a manometric study by Ho and colleagues, at 6 months of follow-up, patients undergoing stapled anastomoses demonstrated significantly reduced resting anal pressures with a higher incidence of endosonographically visible internal anal sphincter fragmentation when compared with patients who did not undergo trans-anal stapled anastomosis [26]. In addition to sphincter damage, neorectal capacity and compliance are central to rectal function. The neorectum, which is part of the descending colon, does not have the same elastic wall properties as the rectum [27]. Therefore, after LAR, the pressure of the anal canal decreases, while the pressure of the neorectum significantly increases even when associated with small fecal volumes.

To further study the precise description of relationship between pelvic parameters and LARS, pelvic dimensions from patients who had an anastomotic level $<5 \mathrm{~cm}$ and underwent surgery more than 18 months were utilized to analysis. Five indicators (based on CT images) were employed in the investigation of pelvic dimensions, including interspinous distance (the narrowest distance between the ischial spines), transverse outlet, sagittal inlet, posterior sagittal diameter of pelvic outlet, and sacrum-coccyx distance. As expected, interspinous diameter of LARS group were significantly lower than no LARS group $(p<0.05)$, while other parameters were not found to be statistically significant. According to previous studies and our own experience, the operative field for LAR is strictly limited transversely by the pelvis at the ischial spine level. In contrast, the anterior-posterior direction of the operative field is relatively more flexible. For example, the organs, such as the bladder, and the prostate or uterus, can be pushed anteriorly to a certain extent.

Our study had several limitations. Several potential risk factors for LARS, including the anastomosis type, point of ligation ("high' or "low") of the inferior mesenteric artery, and obstetric anal sphincter injury, were not prospectively recorded in this study. Due to conservative ideas or extra costs, only $3.6 \%$ of patients were willing to receive presurgery radiation therapy. Another limitation is that the majority of patients were only contacted by telephone and not visited unless they requested it. Despite these limitations, this study has yielded potentially important information about the risk factors of LARS in China.

Author's Contributions The contribution of each author is as follows: $\mathrm{YH}$ and XWD (design the study); QY and PY (data acquisition, analysis, and interpretation); GHD and FWJ (proofread and revise the manuscript); and WS and DGS (collect the date). All authors read and approved the final manuscript.

Funding This work was supported by the Joint Project Sponsored by the Science and Technology Commission and the Health Development Planning Commission of Chongqing for Yuan Qiu (2019QNXM019).

\section{Compliance with Ethical Standards}

Conflict of Interest The authors declare that they have no conflict of interest.

Open Access This article is licensed under a Creative Commons Attribution 4.0 International License, which permits use, sharing, adaptation, distribution and reproduction in any medium or format, as long as you give appropriate credit to the original author(s) and the source, 
provide a link to the Creative Commons licence, and indicate if changes were made. The images or other third party material in this article are included in the article's Creative Commons licence, unless indicated otherwise in a credit line to the material. If material is not included in the article's Creative Commons licence and your intended use is not permitted by statutory regulation or exceeds the permitted use, you will need to obtain permission directly from the copyright holder. To view a copy of this licence, visit http://creativecommons.org/licenses/by/4.0/.

\section{References}

1. Gu J, Chen N (2013) Current status of rectal cancer treatment in China. Color Dis 15(11):1345-1350

2. Emmertsen KJ, Laurberg S (2012) Low anterior resection syndrome score: development and validation of a symptom-based scoring system for bowel dysfunction after low anterior resection for rectal cancer. Ann Surg 255(5):922-928

3. Juul T, Ahlberg M, Biondo S, Espin E, Jimenez LM, Matzel KE, Palmer GJ, Sauermann A, Trenti L, Zhang W, Laurberg S (2014) Christensen. Low anterior resection syndrome and quality of life: an international multicenter study. Dis Colon rectum 57(5):585-591

4. Juul T, Ahlberg M, Biondo S, Emmertsen KJ, Espin E, Jimenez LM, Matzel KE, Palmer G, Sauermann A, Trenti L, Zhang W, Laurberg S, Christensen P (2014) International validation of the low anterior resection syndrome score. Ann Surg 259(4):728-734

5. Hou XT, Pang D, Lu Q, Yang P, Jin SL, Zhou YJ, Tian SH (2015) Validation of the Chinese version of the low anterior resection syndrome score for measuring bowel dysfunction after sphincterpreserving surgery among rectal cancer patients. Eur J Oncol Nurs 19(5):495-501

6. Lin YH, Chen HP, Liu KW (2015) Fecal incontinence and quality of life in adults with rectal cancer after lower anterior resection. J Wound Ostomy Continence Nurs 42(4):395-400

7. Ridolfi TJ, Berger N, Ludwig KA (2016) Low anterior resection syndrome: current management and future directions. Clin Colon Rectal Surg 29(3):239-245

8. Hughes DL, Cornish J, Morris C, Trial Management LARRIS (2017) Functional outcome following rectal surgery-predisposing factors for low anterior resection syndrome. Int J Color Dis 32(5): 691-697. https://doi.org/10.1007/s00384-017-2765-0

9. Bryant CL, Lunniss PJ, Knowles CH, Thaha MA, Chan CL (2012) Anterior resection syndrome. Lancet Oncol 13(9):403-408

10. K. J. Emmertsen, S. Laurberg, and G (2013) Rectal cancer function study. Impact of bowel dysfunction on quality of life after sphincter-preserving resection for rectal cancer. Br J Surg 100(10): 1377-1387

11. T. Y. Chen, L. M. Wiltink, R. A. Nout, (2015) Meershoek-Klein Kranenbarg E, S. Laurberg, C. A. Marijnen, and C. J. van de Velde. Bowel function 14 years after preoperative short-course radiotherapy and total mesorectal excision for rectal cancer: report of a multicenter randomized trial. Clin Colorectal Cancer 14(2): 106-114

12. Bregendahl S, Emmertsen KJ, Lous J, Laurberg S (2013) Bowel dysfunction after low anterior resection with and without neoadjuvant therapy for rectal cancer: a population-based cross-sectional study. Color Dis 15(9):1130-1139

13. P. Ekkarat, T. Boonpipattanapong, K. Tantiphlachiva, and S. Sangkhathat (2016) Factors determining low anterior resection syndrome after rectal cancer resection: a study in Thai patients. Asian J Surg 2016; 39(4): 225-231

14. Jimenez-Rodriguez RM, Segura-Sampedro JJ, Rivero-Belenchon I, Diaz Pavon JM, Garcia Cabrera AM, Vazquez Monchul JM, Padillo J, de la Portilla F (2017) Is the interval from surgery to ileostomy closure a risk factor for low anterior resection syndrome? Color Dis 19(5):485-490

15. Ho P, Law WL, Chan SC, Lam CK, Chu KW (2003) Functional outcome following low anterior resection with total mesorectal excision in the elderly. Int J Color Dis 18(3):230-233

16. Bauerle T, Seyler L, Munter M, Jensen A, Brand K, Fritzsche KH, Kopp-Schneider A, Schussler M, Schlemmer HP, Stieltjes B, Ganten M (2013) Diffusion-weighted imaging in rectal carcinoma patients without and after chemoradiotherapy: a comparative study with histology. Eur J Radiol 82(3):444-452

17. Jimenez-Gomez LM, Espin-Basany E, Trenti L, Marti-Gallostra M, Sanchez-Garcia JL, Vallribera-Valls F, Kreisler E, Biondo S, Armengol-Carrasco M (2018) Factors associated with low anterior resection syndrome after surgical treatment of rectal cancer. Color Dis 20(3):195-200

18. Scheer AS, Boushey RP, Liang S, Doucette S, O'Connor AM, Moher D (2011) The long-term gastrointestinal functional outcomes following curative anterior resection in adults with rectal cancer: a systematic review and meta-analysis. Dis Colon rectum 54(12):1589-1597

19. Denost Q, Laurent C, Capdepont M, Zerbib F, Rullier E (2011) Risk factors for fecal incontinence after intersphincteric resection for rectal cancer. Dis Colon rectum 54(8):963-968

20. Liu LG, Yan XB, Shan ZZ, Yan LL, Jiang CY, Zhou J, Tian Y, Jin ZM (2017) Anorectal functional outcome following laparoscopic low anterior resection for rectal cancer. Mol Clin Oncol 6(4):613621

21. Rubin F, Douard R, Wind P (2014) The functional outcomes of coloanal and low colorectal anastomoses with reservoirs after low rectal cancer resections. Am Surg 12:1222-1229

22. Ziv Y, Zbar A, Bar-Shavit Y, Igov I (2013) Low anterior resection syndrome (LARS): cause and effect and reconstructive considerations. Tech Coloproctol 17(2):151-162

23. Y. C. Zhang, X. D. Jin, Y. T. Zhang, and Z. Q (2012) Wang. Better functional outcome provided by short-armed sigmoid colon-rectal side-to-end anastomosis after laparoscopic low anterior resection: a match-paired retrospective study from China. Int J Color Dis 27(4): 535-541

24. O'Riordain MG, Molloy RG, Gillen P, Horgan A, Kirwan WO (1992) Rectoanal inhibitory reflex following low stapled anterior resection of the rectum. Dis Colon rectum 35(9):874-878

25. Horgan AF, Molloy RG, Coulter J, Sheehan M, Kirwan WO (1993) Nerve regeneration across colorectal anastomoses after low anterior resection in a canine model. Int J Color Dis 8(3):167-169

26. Ho YH, Tsang C, Tang CL, Nyam D, Eu KW, Seow-Choen F (2000) Anal sphincter injuries from stapling instruments introduced transanally: randomized, controlled study with endoanal ultrasound and anorectal manometry. Dis Colon rectum 43(2):169-173

27. Liang Z, Ding W, Chen W, Wang Z, Du P, Cui L (2016) Therapeutic evaluation of biofeedback therapy in the treatment of anterior resection syndrome after sphincter-saving surgery for rectal cancer. Clin Colorectal Cancer 15(3):101-107

Publisher's Note Springer Nature remains neutral with regard to jurisdictional claims in published maps and institutional affiliations. 